\title{
A Design Method of Precise Positioning Stage in Vacuum Environment by Disturbance Observer with Position Dependent Parameter
}

\author{
KazUhiro Morita $^{* \dagger}$ Member, $\quad$ ShinjI WAKUI ${ }^{\ddagger}$ Non-member
}

(Received July 15, 2019, revised October 4, 2019)

\begin{abstract}
The semiconductor inspection apparatus using an electron beam in the vacuum environment requires high resolution and measuring accuracy because the semiconductor devices which are smaller and thinner are required by growing market of the smartphone and the tablet PC. Herewith, high positioning accuracy of XY stage working in vacuum environment is also required. However, it is hard to introduce the aerostatic bearing like semiconductor photolithography apparatus into vacuum environment due to technical difficulties and cost. Therefore, friction drive type XY stage was adopted. Nevertheless, it is difficult to precisely control and adjust characteristics and behavior as for friction drive $\mathrm{XY}$ stage which is working in vacuum environment. The characteristic of $\mathrm{XY}$ stage fluctuates in vacuum environment even though it has been precisely adjusted in the atmosphere. Moreover, nobody can re-adjust mechanical characteristics unless they open a vacuum chamber. It means an electron beam is halted, and the long time is necessary to restart emitting an electron beam. Therefore, this paper proposes a design approach which is useful for high precision positioning of $\mathrm{XY}$ stage in vacuum environment by the feedback of estimated disturbance which includes position dependent parameter. Position dependent parameter is determined by measuring evaluation function value at each position. Furthermore, proposed design method is also useful to maintain performance for aged deterioration. In this paper, issue of XY stage is mentioned at first. Then, a design approach which comprises a disturbance observer including position dependent parameter and state feedback is described. At last, experiment results are shown.
\end{abstract}

Keywords: Precision positioning, Vacuum environment, Disturbance observer, Feedback control, Semiconductor device inspection, Electron beam

\section{Introduction}

High integrated semiconductor device is advancing by the demand of smartphone, tablet PC and IoT. According to the ITRS (International Technology Roadmap for Semiconductor), a design rule will become $10 \mathrm{~nm}$ in 2020 [1]. CD-SEM (Critical Dimension Scanning Electron-beam Microscope) is used for the inspection of these semiconductor devices [2]. It has a trait that inspects a sample in vacuum environment. Positioning accuracy of XY stage (hereinafter called the stage) less than $3 \mathrm{~nm}$ is required by CD-SEM which inspects circuit pattern less than $10 \mathrm{~nm}$. As well as known, semiconductor photolithography apparatus has been equipped with the stage using aerostatic bearing to get high accuracy [3] [6]. However, it was hard to introduce it into CD-SEM which is working in vacuum environment due to high cost and technical difficulties. Cost

\footnotetext{
* Corresponding author: kazuhiro.morita.fg@hitachi-hightech.com

$\dagger$ Hitachi High Technologies Corp.,

882 Ichige,Hitachinaka-city, Ibaraki, Japan 312-8504

$¥$ Tokyo University of Agriculture and Technology,

2-24-16 Naka-cho, Koganei-shi, Tokyo, Japan, 184-8588
}

reduction is also important theme to manufacture CDSEM. Even though the stage which equipped with high precision ball-screw is low cost, it is difficult to adopt it because it has a decrease in positioning accuracy by heat transmission [7].

By such background, a friction drive type stage using the linear motor of the direct drive has been developed. Its purpose is to manage both cost and positioning accuracy. Nevertheless, it is not easy to control the friction drive type stage precisely and stably because it has position and pressure dependencies. Particularly, it is very serious that the dynamic behavior of the stage fluctuates by pressure changing. The stage does not precisely work in a vacuum environment even though it has been assembled and precisely adjusted in the atmosphere workspace. Moreover, the stage cannot maintain stability in the whole movable area by a conventional controller.

Therefore, this paper proposes a controller design method to substantiate high-precision and stable positioning in a vacuum environment. This paper comprises three parts. At first, an issue of a friction drive 


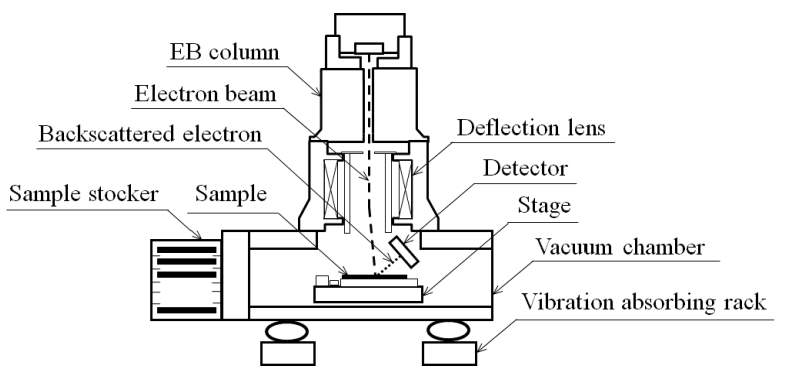

Figure 1: Structure of CD-SEM.

type stage in a vacuum environment is clarified. Secondly, a controller design method using the disturbance observer with a position-dependent parameter (hereinafter called PDP) is proposed. Although the disturbance observer is well known [8] [11], there were not application cases using it for high-precision positioning in a vacuum environment. Moreover, the stage has to work in the whole movable area stably. Thus, this paper proposes a design method that precisely and stably controls the positioning stage working in a vacuum environment by PDP based on actual measurement. This proposed method is similar to a well-known disturbance observer-based control [12]. In the conventional method, an output filter used for stabilization has been implemented in the inner disturbance attenuation loop like this proposed method. Such an output filter is almost designed as a low pass filter based on the transfer function. However, the output filter is not effective for the stage working in a vacuum environment because the filter does not consider position and pressure dependencies. In this paper, PDP is determined by actual measurement because the parameter varying is not able to determine analytically. Thus, this proposed method is a practical method that has been combined with the state feedback control and a disturbance observer (hereinafter called DOB) including PDP. Finally, the experimental results of the proposed design method are shown.

\section{Positioning stage in vacuum environment}

In this chapter, an issue of a friction drive type stage working in vacuum environment is clarified by showing a structure and its characteristics.

\subsection{Structure of CD-SEM and positioning stage}

Fig. 1 shows the structure of the CD-SEM. There are samples to be measured into a sample stocker. Then, a sample is carried into a vacuum chamber (hereinafter called the chamber). There is a stage in the chamber. The sample is loaded onto the stage. After that, the sample changes the measurement position by moving the stage. An electron beam that has been emitted from the electron beam column is deflected by an electrical deflection lens. A circuit pattern formed on the sample is irradiated with the electron beam. An image is generated by a detector that detects a backscattered electron that has been emitted from the surface of the sample.

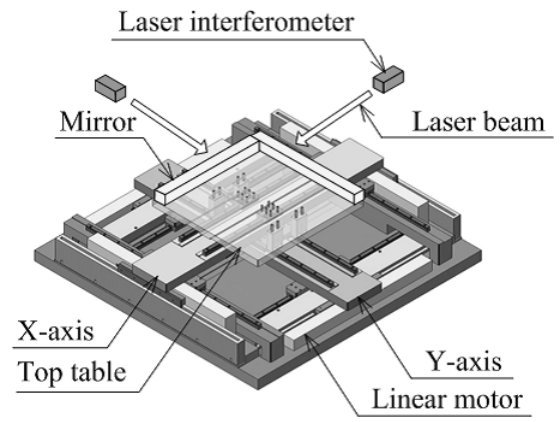

Figure 2: Structure of the $\mathrm{XY}$ stage which is friction drive type.

The circuit pattern is inspected based on an image. A vibration absorber is placed under the chamber to reduce vibration. This paper argues regarding the stage which works in a vacuum environment.

Fig. 2 shows the structure of the stage, which is a friction drive type stage and is placed into the chamber. The stage moves in two axial directions of $\mathrm{x}$ and y. A top table fixing a sample is connected to a linear motor through a guide. Therefore, the top table moves the two-dimensional surface by driving two linear motors. The position of the top table is measured by a laser interferometer and reflection mirror. The stage has a two-dimensional large movable area of 500 square $\mathrm{mm}$ toward both axes of $\mathrm{x}$ and $\mathrm{y}$.

2.2 Characteristics of the XY stage Although the stage is assembled and adjusted in the atmosphere, the stage works in a vacuum environment of 10-5 Pa. A difference in pressure causes a change in the characteristic of the stage. Then, nobody can re-adjust the stage, which is placed in a vacuum environment, even if characteristic change can be found. The chamber should be opened when engineers re-adjust the stage because they have to handle and adjust the stage in the atmosphere. In addition, opening the chamber means that an electron beam should be halted, and a long time is necessary to restart emitting an electron beam.

Particularly, a pressure change causes the change of friction characteristics. Fig. 3 shows an open-loop Bode diagram to compare the frequency response in the atmosphere and vacuum environment. According to the gain diagram, it has shown the second-order lag element properties regarding the atmosphere. However, it seems to have been reduced friction and an elastic element in a vacuum environment. Moreover, a resonance point has appeared to the frequency of $450 \mathrm{~Hz}$ in a vacuum environment. According to the phase diagram, the phase lag in the vacuum environment has become larger more than that in the atmosphere. In addition, there are the position-dependent properties in vacuum environment even though the engineer has adjusted the stage precisely in the atmosphere. Fig. 4 shows five Bode diagrams that were measured at each position in a vacuum environment. Five points are lo- 


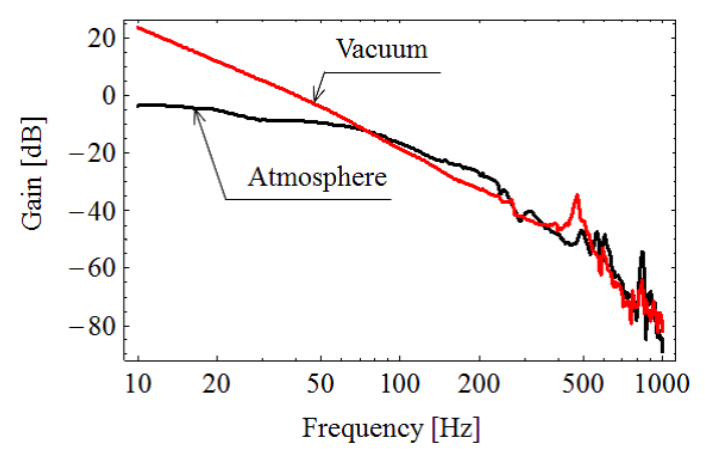

(a) Gain diagram

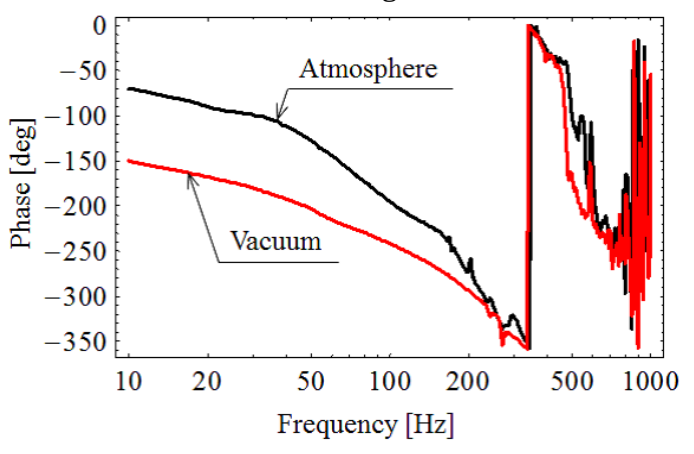

(b) Phase diagram

Figure 3: Bode diagram to compare the vacuum environment with the atmosphere.

cated at $(250 \mathrm{~mm}, 250 \mathrm{~mm}),(30 \mathrm{~mm}, 30 \mathrm{~mm}),(470 \mathrm{~mm}$, $470 \mathrm{~mm}),(30 \mathrm{~mm}, 470 \mathrm{~mm})$ and $(470 \mathrm{~mm}, 30 \mathrm{~mm})$. The position $(250 \mathrm{~mm}, 250 \mathrm{~mm})$ is the center of the moveable area. Blackline in Fig. 4 shows the Bode diagram, which has been measured at the position ( $250 \mathrm{~mm}, 250$ $\mathrm{mm})$. The red line shows the diagram at $(30 \mathrm{~mm}, 30$ $\mathrm{mm})$. The green line shows the diagram at $(30 \mathrm{~mm}, 470$ $\mathrm{mm})$. The yellow line shows the diagram at $(470 \mathrm{~mm}$, $30 \mathrm{~mm})$. The blue line shows the diagram at $(470 \mathrm{~mm}$, $470 \mathrm{~mm})$. According to the gain diagram, it seems to be the second-order lag element at $470 \mathrm{~mm}$ of $\mathrm{x}$ axis. Gain characteristics are also affected by the position of y axis. Phase diagrams which were measured at each position also show the position dependency. The resonance frequency is different in each position. However, it is hard to precisely adjust the stage in a vacuum environment because the engineer cannot handle the stage. For such reasons, it is difficult to substantiate precise positioning in the vacuum environment, even though the controller and its parameter were precisely determined in the atmosphere. Furthermore, it is difficult to maintain stability in the whole moveable area by the controller and its parameters, which were determined in the atmosphere.

\section{Disturbance observer with feedback gain de- pends on the stage position}

In this chapter, a controller design method is proposed. The method comprises the disturbance observer and PDP. At first, a model of the stage is given. After that,

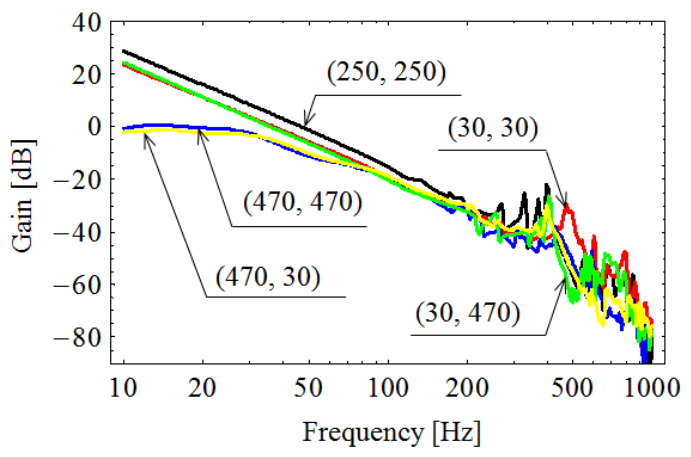

(a) Gain diagram

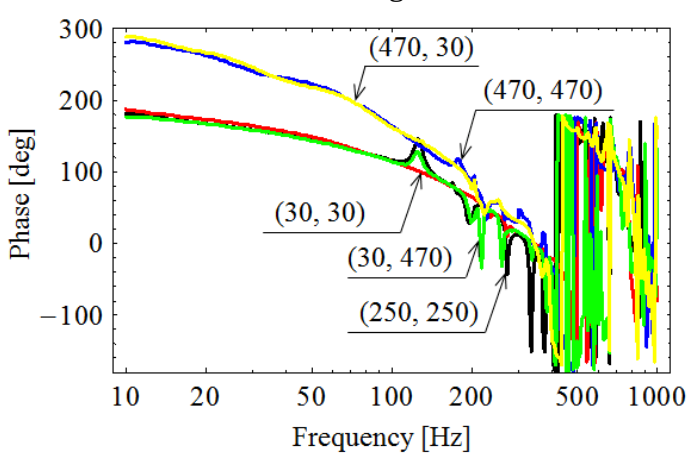

(b) Phase diagram

Figure 4: Open loop Bode diagram at each position in the vacuum environment.

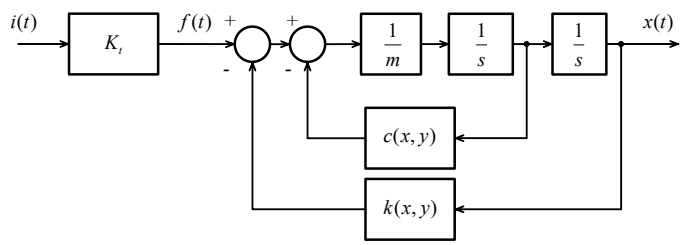

Figure 5: Block diagram of the $\mathrm{XY}$ stage limited to $\mathrm{x}$ axis.

a controller design method is mentioned based on that stage model. This paper argues only $\mathrm{x}$-axis of the stage because the method of $y$-axis is the same as $\mathrm{x}$-axis.

3.1 Model of the XY stage According to mention in section 2.2 , it was clarified that the stage varies its characteristic in vacuum environment. In this section, a model of the stage is given. At first, a nominal model of the stage is given as an inertial model. Fig. 5 shows a block diagram of the stage limited to $x$-axis. Here, $s$ is Laplace operator. The thrust $f(t)[\mathrm{N}]$ is proportional to driving current $i(t)[\mathrm{A}] . K_{t}$ [N/A] is thrust constant number. $m[\mathrm{~kg}]$ is mass of $x$-axis. Here, $k(x, y)[\mathrm{N} / \mathrm{m}]$ is an elastic coefficient. $c(x, y)[\mathrm{Ns} / \mathrm{m}]$ is a coefficient of viscosity. $x(t)[\mathrm{m}]$ is displacement of $x$-axis from the origin position. $y(t)[\mathrm{m}]$ is displacement of $y$-axis from the origin position. $k(x, y)$ and $c(x, y)$ have position dependency. Equation (1) which is an equation of motion of the stage is given by Fig. 5 .

$$
K_{t} \dot{i}(t)=m \ddot{x}(t)+c(x, y) \dot{x}(t)+k(x, y) x(t)
$$




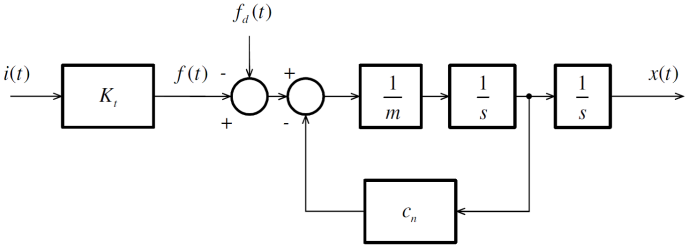

Figure 6: Block diagram of the XY stage with disturbance.

Table 1: Physical parameter of the XY stage measured in the atmosphere.

\begin{tabular}{llll}
\hline Parameter & Description & Value & Unit \\
\hline$m$ & $\begin{array}{l}\text { Mass of x-axis including } \\
\text { the top table }\end{array}$ & 46.8 & $\mathrm{~kg}$ \\
$c_{n}$ & $\begin{array}{l}\text { Nominal coefficient of vis- } \\
\text { cosity }\end{array}$ & 5.0 & $\mathrm{Ns} / \mathrm{m}$ \\
$K_{t}$ & Thrust constant & 242.2 & $\mathrm{~N} / \mathrm{A}$ \\
\hline
\end{tabular}

However, as for equation (1), it is difficult to determine $c(x, y)$ and $k(x, y)$ because they are changing during moving the stage. Accordingly, the stage model has been supposed that the nominal stage model has been varied its properties by the disturbance. For that reason, $c(x, y)$ and $k(x, y)$ mean that $c_{n}$ and $k_{n}$ have been changed by the disturbance to $c_{n}+\Delta c(t, x, y)$ and $k_{n}+\Delta k(t, x, y)$. Here, $c_{n}[\mathrm{Ns} / \mathrm{m}]$ is nominal coefficient of viscosity, and $k_{n}[\mathrm{~N} / \mathrm{m}]$ is nominal elastic coefficient. Coefficients $c_{n}$ and $k_{n}$ have been measured as nominal value in the atmosphere because it is difficult to measure them in vacuum environment. The stage model can transform into equation (2) when $f_{d}(t)[\mathrm{N}]$ was defined as disturbance force. Here, disturbance force $f_{d}(t)$ is given as equation (3). $k_{n} x(t)$ in equation (3) has been supposed as a part of disturbance because it can suppose that it is very small in vacuum environment by measured Bode diagram. On the other hand, $c_{n}$ in equation (2) should be left even if the stage is placed in vacuum environment because the stage is friction drive type stage. Moreover, $f_{d}(t)$ is defined as equation (4). It means the disturbance is zeroth order hold.

$$
\begin{aligned}
& K_{t} i(t)=m \ddot{x}(t)+c_{n} \dot{x}(t)+f_{d}(t) \\
& f_{d}(t)=\Delta c(t, x, y) \dot{x}(t)+\left\{k_{n}+\Delta k(t, x, y)\right\} x(t) \\
& \dot{f}_{d}(t)=0
\end{aligned}
$$

As a result, the block diagram of the stage model based on equation (2) is given as Fig. 6 . Table 1 shows physical parameters even though they have been measured in the atmosphere. State equation of equation (5) is given based on equations (2) and (4). It is linear time invariant system. Since the observability matrix of equation (5) has rank=3, this system is observable.

$$
\begin{aligned}
& \dot{\boldsymbol{X}}(t)=\boldsymbol{A} \boldsymbol{X}(t)+\boldsymbol{B} i(t) \\
& x(t)=\boldsymbol{C X}(t)
\end{aligned}
$$

Here, $\boldsymbol{X}(t), \boldsymbol{A}, \boldsymbol{B}, \boldsymbol{C}$ are as follows.

$$
\begin{aligned}
& \boldsymbol{X}(t)=\left[\begin{array}{c}
x(t) \\
\dot{x}(t) \\
f_{d}(t)
\end{array}\right], \quad \boldsymbol{A}=\left[\begin{array}{ccc}
0 & 1 & 0 \\
0 & -\frac{c_{n}}{m} & -\frac{1}{m} \\
0 & 0 & 0
\end{array}\right], \quad \boldsymbol{B}=\left[\begin{array}{c}
0 \\
\frac{K_{t}}{m} \\
0
\end{array}\right], \\
& \boldsymbol{C}=\left[\begin{array}{lll}
0 & 0 & 0
\end{array}\right],
\end{aligned}
$$

3.2 A design method using disturbance observer with position dependent parameter This section is comprised of three subsections. In the first subsection, a design of DOB and cancelling its disturbance are described. In the second subsection, a way to determine PDP is mentioned. In the third subsection, a state feedback controller of servo system is described. This design method has advantages of including PDP in feedback gain of estimated disturbance, and of determining PDP based on actual measurement.

3.2.1 Disturbance observer The matrix $\boldsymbol{X}(t)$ which is including disturbance $f_{d}(t)$ can be estimated by the full order estimator (hereinafter called FOE) of Luenberger [13]. It is the feedback control model of the output error to get $\hat{\boldsymbol{X}}(t)$ that is the estimated $\boldsymbol{X}(t)$. That model is shown in equation (6). Here, $\boldsymbol{L}$ is feedback gain matrix.

$$
\dot{\hat{\boldsymbol{X}}}(t)=(\boldsymbol{A}-\boldsymbol{L} \boldsymbol{C}) \hat{\boldsymbol{X}}(t)+\boldsymbol{B} i(t)+\boldsymbol{L} x(t)
$$

Here, $\hat{x}^{\mathrm{T}}(t)=\left[\begin{array}{lll}\hat{x}(t) & \hat{\dot{x}} & f_{d}(t)\end{array}\right]$.

In this study, well known LQ (Linear Quadratic) optimal control method has been chosen to determine the feedback gain matrix $\boldsymbol{L}$. The method is able to keep robustness. Equation (7) which is a quadratic form evaluation function has been defined as an evaluation function of the LQ optimal control method. In equation (7), $\boldsymbol{Q}$ is a positive semidefinite matrix and $R$ is a positive number. Then, feedback gain matrix $\boldsymbol{L}$ to minimize evaluation function $J$ is given by $\boldsymbol{L}^{\mathrm{T}}=R^{-1} \boldsymbol{C P}$. Here, $\boldsymbol{P}$ is the unique positive definite matrix satisfying the Riccati equation [14].

$$
J=\int_{0}^{\infty}\left\{\hat{\boldsymbol{X}}^{\mathrm{T}}(t) \boldsymbol{Q} \hat{\boldsymbol{X}}(t)+R i^{2}(t)\right\} d t
$$

In this paper, $\boldsymbol{Q}, R, \boldsymbol{L}$ have been determined by computational simulation as follows. Particularly, $\boldsymbol{Q}$ has been determined in consideration of that it can estimate a disturbance force with positioning of the nano meter order $x(t)$.

$$
\boldsymbol{Q}=\left[\begin{array}{ccc}
1 & 0 & 0 \\
0 & 1 & 0 \\
0 & 0 & 10^{27}
\end{array}\right], \quad R=1, \quad \boldsymbol{L}=\left[\begin{array}{c}
1.7550 \times 10^{4} \\
1.5400 \times 10^{8} \\
3.1623 \times 10^{13}
\end{array}\right]
$$

Thus, DOB which is estimating states $x(t), \dot{x}(t)$ and disturbance $f_{d}(t)$ has been designed. The disturbance can be cancelled by feedback of estimated $\hat{f}_{d}(t)$. Practically, DOB is implemented as discrete system because it is calculated by micro-computer. DOB is discretized by the bilinear transform [15]. Sampling period is 100 $\mu \mathrm{s}$ in discrete DOB. 


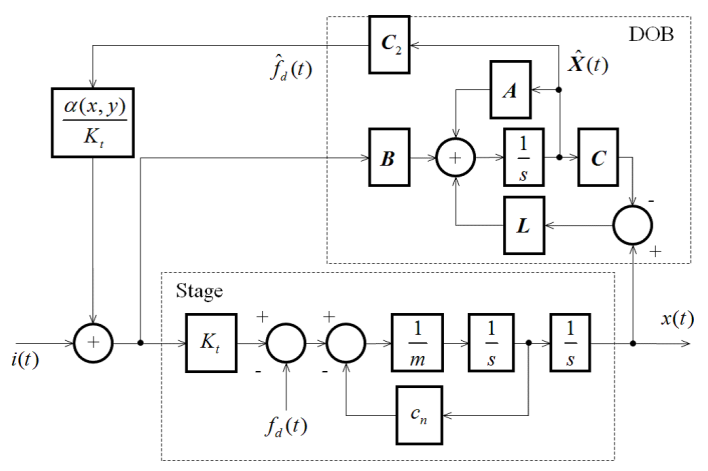

Figure 7: Block diagram of proposed control system which controls the XY stage in vacuum environment using the disturbance observer with position dependent parameter.

On the other hand, the system of equation (5) is uncontrollable because the controllability matrix of equation (5) has rank=2. Therefore, it is impossible to adopt the state feedback control to the stage of equation (5) even though all state variables have been estimated. Hence, an inner feedback control loop has been configured using $\hat{f}_{d}(t)$. The stage should have apparent nominal characteristics by cancelling out disturbance. However, its feedback system has failed because the system could not maintain stability in whole movable area. It could not maintain both stability and positioning accuracy because fluctuation range of the stage characteristics were too large. Hence this paper proposes the feedback control of estimated disturbance including PDP. PDP is determined by actual measuring of effectivity. It is suitable for stage control in vacuum environment even though it is simple. Fig. 7 shows a block diagram of the stage which has been linearized by cancelling disturbance. Here, output matrix $\boldsymbol{C}_{2}$ is $\boldsymbol{C}_{2}=\left[\begin{array}{lll}0 & 0 & 1\end{array}\right]$. In addition, feedback gain $\alpha(x, y)$ is PDP which has been introduced. The gain $\alpha(x, y)$ varies in accordance with position of the stage. It is determined by actual measuring of evaluation function for each position. Detail of $\alpha(x, y)$ is described in subsection 3.2.2.

\subsubsection{Feedback gain depending on the stage po-}

sition In this subsection, a way to determine $\alpha(x, y)$ is described. Feedback gain $\alpha(x, y)$ consists $n \times m$ number of $\alpha\left(x_{i}, y_{j}\right)$. The gain $\alpha\left(x_{i}, y_{j}\right)$ are placed at equal intervals in whole movable area. The gain $\alpha\left(x_{i}, y_{j}\right)$ are determined from $i=1$ to $n$ and from $j=1$ to $m$. The stage moves to the point $\left(x_{1}, y_{1}\right)$. Feedback gain $\alpha\left(x_{1}, y_{1}\right)$ is measured as the most effective coefficient for attenuating disturbance. Then, the stage moves to the next point $\left(x_{1}, y_{2}\right)$, and measures $\alpha\left(x_{1}, y_{2}\right)$. By repeating of moving and measuring, all $\alpha\left(x_{i}, y_{j}\right)$ are measured likewise. Thus, $\alpha\left(x_{1}, y_{1}\right), \alpha\left(x_{1}, y_{2}\right), \ldots, \alpha\left(x_{1}, y_{m}\right), \alpha\left(x_{2}, y_{1}\right)$, $\alpha\left(x_{2}, y_{2}\right), \ldots, \alpha\left(x_{2}, y_{m}\right), \ldots, \alpha\left(x_{n}, y_{m}\right)$ are determined. As for determination of $\alpha\left(x_{i}, y_{i}\right)$, equation (8) was used as evaluation function $V_{i j}$. Evaluation function $V_{i j}$ is calculated at each position $\left(x_{i}, y_{j}\right)$. Here, $\Delta T$ [s] is sampling period to calculate $V_{i j}$. Variable $I_{t}$ is the number

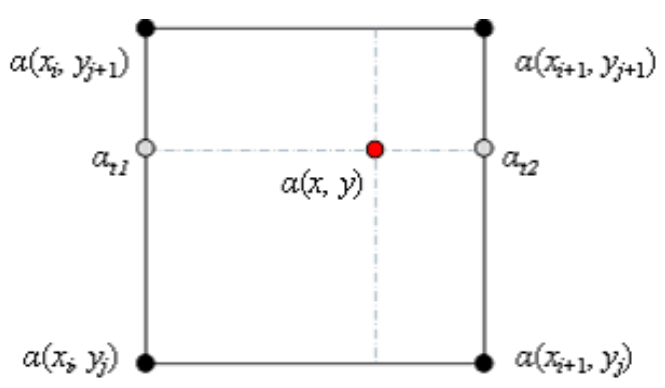

Figure 8: Interpolating $\alpha(x, y)$ by bilinear interpolation method.

of calculations. Evaluation function $V_{i j}$ should be the least numerical value when $\alpha\left(x_{i}, y_{j}\right)$ is the most effective.

$$
V_{i j}=\sum_{k=0}^{I_{t}}\left\{\left(x_{i}-x(k \cdot \Delta T)\right)^{2}+\left(y_{i}-y(k \cdot \Delta T)\right)^{2}\right\}
$$

Feedback gain $\alpha\left(x_{i}, y_{j}\right)$ are discretely placed even though the stage moves continuously. Therefore, $\alpha\left(x_{i}, y_{j}\right)$ should be interpolated in real time while the stage is moving. The bilinear interpolation method has been adopted to interpolate $\alpha(x, y)$ even though the method is used in many field of image processing [16] [18]. At this time, $\alpha(x, y)$ should be interpolated three-dimensional. Fig. 8 shows a way of threedimensional interpolation. At first, four points $\left(x_{i}, y_{j}\right)$, $\left(x_{i+1}, y_{j}\right),\left(x_{i}, y_{j+1}\right),\left(x_{i+1}, y_{j+1}\right)$ around $(x, y)$ are found. Then, $\alpha_{t 1}$ and $\alpha_{t 2}$ are calculated by equations (9) and (10). Here, $w_{x}$ is $\left(x_{i+1}-x\right) /\left(x_{i+1}-x_{i}\right), w_{y}$ is $\left(y_{j+1}-\right.$ $y) /\left(y_{j+1}-y_{j}\right)$. After that, $\alpha(x, y)$ can be interpolated by equation (11). Thus, position dependent feedback gain $\alpha(x, y)$ is determined.

$$
\begin{aligned}
& \alpha_{t 1}=\left(1-w_{y}\right) \alpha\left(x_{i}, y_{j}\right)+w_{y} \alpha\left(x_{i}, y_{j+1}\right) \\
& \alpha_{t 2}=\left(1-w_{y}\right) \alpha\left(x_{i+1}, y_{j}\right)+w_{y} \alpha\left(x_{i+1}, y_{j+1}\right) \\
& \alpha(x, y)=\left(1-w_{x}\right) \alpha_{t 1}+w_{x} \alpha_{t 2}
\end{aligned}
$$

3.2.3 State feedback control The disturbance which varies the stage characteristics has been canceled by feedback including PDP. Thus, the disturbance can be ignored. In this subsection, the state feedback control for precision positioning is mentioned. An integral compensator has been adopted to make servo positioning system of the type 1. Equation (12) shows an equation of an integral compensator.

$$
\dot{e}(t)=r(t)-x(t)
$$

Moreover, estimated state variable $\hat{\hat{x}}(t)$ can be used instead of variable $\dot{x}(t)$. Then, equation (13) is given by removing the disturbance from equation (5), and appending an integral compensator. This system is controllable because controllable matrix has rank $=3$. The state feedback controller which should be a servo positioning system comprises a state feedback control and 
an integral compensator.

$$
\begin{aligned}
& \dot{\boldsymbol{X}}_{\boldsymbol{e}}(t)=\boldsymbol{A}_{\boldsymbol{e}} \boldsymbol{X}_{\boldsymbol{e}}(t)+\boldsymbol{B}_{\boldsymbol{e}} i(t)+\boldsymbol{v}(t) \\
& x(t)=\boldsymbol{C}_{\boldsymbol{e}} \boldsymbol{X}_{\boldsymbol{e}}(t)
\end{aligned}
$$

Here, $\boldsymbol{X}_{\boldsymbol{e}}(t), \boldsymbol{A}_{\boldsymbol{e}}, \boldsymbol{B}_{\boldsymbol{e}}, \boldsymbol{C}_{\boldsymbol{e}}, \boldsymbol{v}(t)$ are as follows.

$$
\begin{aligned}
& \boldsymbol{X}_{\boldsymbol{e}}(t)=\left[\begin{array}{c}
x(t) \\
\hat{\dot{x}}(t) \\
e(t)
\end{array}\right], \quad \boldsymbol{A}_{\boldsymbol{e}}=\left[\begin{array}{ccc}
0 & 1 & 0 \\
0 & -\frac{c_{n}}{m} & 0 \\
1 & 0 & 0
\end{array}\right], \quad \boldsymbol{B}_{\boldsymbol{e}}=\left[\begin{array}{c}
0 \\
\frac{K_{t}}{m} \\
0
\end{array}\right], \\
& \boldsymbol{C}_{\boldsymbol{e}}=\left[\begin{array}{lll}
1 & 0 & 0
\end{array}\right], \quad \boldsymbol{v}(t)=\left[\begin{array}{lll}
0 & 0 & r(t)
\end{array}\right]
\end{aligned}
$$

Equation (14) shows the control law. A matrix $K_{f}$ is the state feedback gain matrix. An element $K_{p}$ is scalar integral gain for servo positioning system.

$$
i(t)=-\boldsymbol{K}_{\boldsymbol{f}} \boldsymbol{X}_{\boldsymbol{e}}(t)
$$

Here, $\boldsymbol{K}_{\boldsymbol{f}}$ is as follow.

$$
\boldsymbol{K}_{\boldsymbol{f}}=\left[\begin{array}{lll}
k_{11} & k_{12} & -K_{p}
\end{array}\right]
$$

Moreover, closed loop state equation is given as equation (15) is based on equations (13) and (14). According to equation (15), steady state positional error $e(\infty)$ will be clearly zero if the closed loop system is stable.

$$
\dot{\boldsymbol{X}}_{\boldsymbol{e}}(t)=\left(\boldsymbol{A}_{\boldsymbol{e}}-\boldsymbol{B}_{\boldsymbol{e}} \boldsymbol{K}_{\boldsymbol{f}}\right) \boldsymbol{X}_{\boldsymbol{e}}(t)+\boldsymbol{v}(t)
$$

LQ optimal control method has been adopted to determine state feedback gain matrix $\boldsymbol{K}_{\boldsymbol{f}}$. Equation (16) which is a quadratic form equation is defined as an evaluation function of the LQ optimal control method. In equation (16), $\boldsymbol{Q}_{\boldsymbol{e}}$ and $R_{e}$ are the weighting matrices. The matrix $\boldsymbol{Q}_{\boldsymbol{e}}$ is a positive semidefinite matrix and $R_{e}$ is a positive number. Then, the gain matrix $\boldsymbol{K}_{\boldsymbol{f}}$ is given by $\boldsymbol{K}_{\boldsymbol{f}}=R_{e}^{-1} \boldsymbol{B}_{\boldsymbol{e}}^{\mathrm{T}} \boldsymbol{P}_{\boldsymbol{e}}$. It is the minimize an evaluation function $J_{e}$. Here, $\boldsymbol{P}_{\boldsymbol{e}}$ is the unique positive definite matrix satisfying the Riccati equation of equation (17).

$$
\begin{aligned}
& J_{e}=\int_{0}^{\infty}\left\{\boldsymbol{X}_{\boldsymbol{e}}^{\mathrm{T}}(t) \boldsymbol{Q}_{\boldsymbol{e}} \boldsymbol{X}_{\boldsymbol{e}}(t)+R_{e} i^{2}(t)\right\} d t \\
& \boldsymbol{P}_{\boldsymbol{e}} \boldsymbol{A}_{\boldsymbol{e}}+\boldsymbol{A}_{\boldsymbol{e}}^{\mathrm{T}} \boldsymbol{P}_{\boldsymbol{e}}-\boldsymbol{P}_{\boldsymbol{e}} \boldsymbol{B}_{\boldsymbol{e}} R_{e}^{-1} \boldsymbol{B}_{\boldsymbol{e}}^{\mathrm{T}} \boldsymbol{P}_{\boldsymbol{e}}+\boldsymbol{Q}_{\boldsymbol{e}}^{\mathrm{T}}=0
\end{aligned}
$$

The feedback gain matrix $\boldsymbol{K}_{\boldsymbol{f}}$ has been chosen to be suitable positioning accuracy for a CD-SEM. In this study, $\boldsymbol{Q}_{\boldsymbol{e}}, R_{e}$, and $\boldsymbol{K}_{\boldsymbol{f}}$ have been determined as follows by computational simulation.

$$
\begin{aligned}
\boldsymbol{Q}_{e} & =\left[\begin{array}{ccc}
1 & 0 & 0 \\
0 & 1 & 0 \\
0 & 0 & 10^{20}
\end{array}\right], \quad R_{e}=1, \\
\boldsymbol{K}_{\boldsymbol{f}} & =\left[\begin{array}{lll}
5.3683 \times 10^{6} & 1.4406 \times 10^{3} & -1.0 \times 10^{9}
\end{array}\right]
\end{aligned}
$$

In addition, the stability is maintained in whole moveable area by introducing $\alpha(x, y)$. Fig. 9 shows a block diagram of the proposed system. Here, the output matrix $\boldsymbol{C}_{\mathbf{3}}$ is $\boldsymbol{C}_{\mathbf{3}}=\left[\begin{array}{lll}0 & 1 & 0\end{array}\right]$. This feedback controller is discretized by the bilinear transform. Sampling period is $100 \mu \mathrm{s}$ that is the same as DOB.

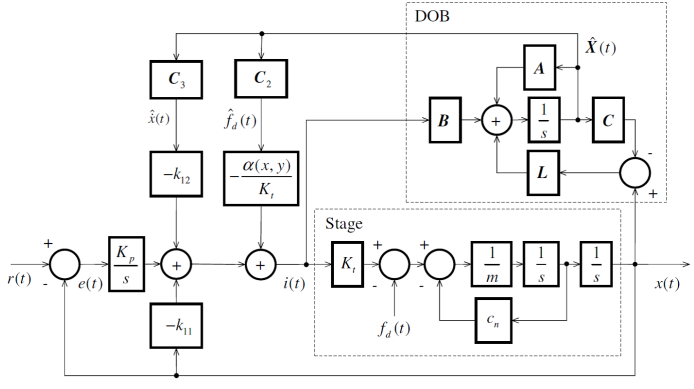

Figure 9: Block diagram of proposed control system which controls the $\mathrm{XY}$ stage in vacuum environment using the disturbance observer with position dependent parameter.

\section{Results of an experiment}

In this chapter, experiment results by proposed design method are shown. At first, measured $\alpha(x, y)$ which is PDP is shown. Then, comparison of Bode diagrams are shown. After that, positioning accuracy is shown.

\subsection{Measurement of position dependent param-} eter $\alpha(\mathbf{x}, \mathbf{y}) \quad$ Fig. 10 shows a map of measured $\alpha\left(x_{i}, y_{j}\right)$. Feedback gain $\alpha\left(x_{i}, y_{j}\right)$ have been measured at each position of the $25 \mathrm{~mm}$ interval. Fig. 10(a) shows a surface plot of $\alpha\left(x_{i}, y_{j}\right)$ at each position, and Fig. 10(b) shows its contour plot. According to these figures, $\alpha\left(x_{i}, y_{j}\right)$ are low around the central area. On the other hand, $\alpha\left(x_{i}, y_{j}\right)$ are high at edge of the stage. The minimum $\alpha\left(x_{i}, y_{j}\right)$ is 0.6 at the central area. The maximum $\alpha\left(x_{i}, y_{j}\right)$ is 2.55 at edge of the stage. It means that much feedback of estimated disturbance is necessary at edge of the stage than central area. By this measuring, it has shown that $\alpha(x, y)$ indicates the position dependency of the stage.

4.2 Frequency characteristics In this section, attenuating effect of the characteristics fluctuation is shown. Fig. 11 shows open loop Bode diagrams of the stage. These diagrams include the feedback loop of estimated disturbance with PDP. Fig. 11(a) shows gain plot. Fig. 11(b) shows phase plot. Its system is equivalent to a block diagram of Fig. 12. It shows that the stage has been linearized by canceling disturbance $f_{d}(t)$. In fact, according to Fig. 11(a), disturbance $f_{d}(t)$ is canceled by the feedback of estimated disturbance $\hat{f}_{d}(t)$ instead of actual $f_{d}(t)$. In Fig. 11(a), diagrams are almost same even though they have been measured at different position. It means that the feedback of estimated disturbance with PDP is effective to control the stage in vacuum environment. Proposed design method has stabilized it in whole moveable area even though conventional simple feedback of estimated disturbance has not been able to stabilize the stage.

4.3 Positioning accuracy In this section, positioning accuracy during steady state by proposed method is mentioned. Fig. 13 shows three positioning trends in the steady state at different position. Each di- 


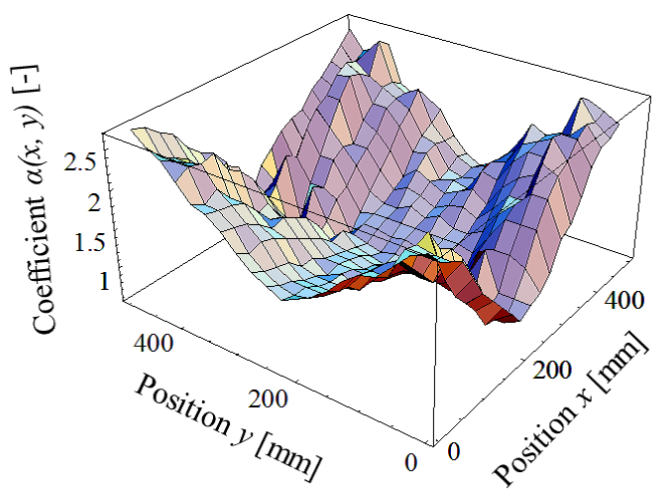

(a) Surface plot of coefficient $\alpha\left(x_{i}, y_{i}\right)$

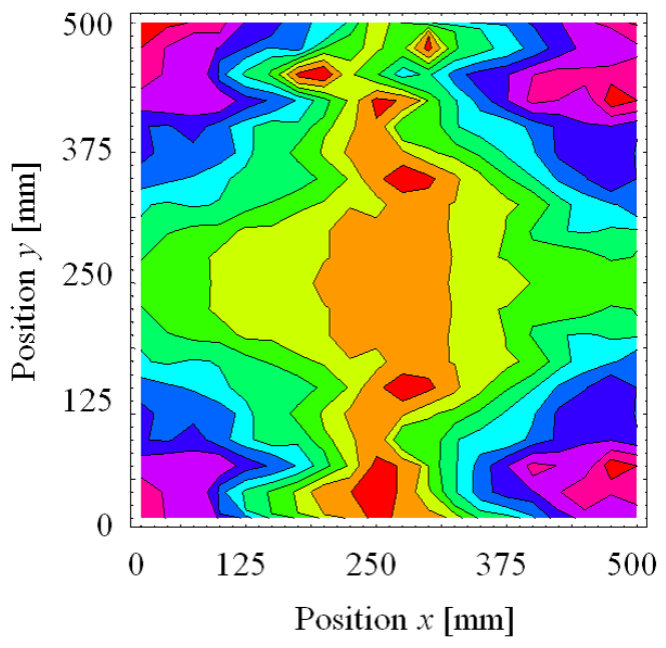

(b) Contour plot of coefficient $\alpha\left(x_{i}, y_{i}\right)$

Figure 10: Measured position dependent parameter $\alpha(x, y)$ at each position.

agram has two trend lines. They are comparison with having the feedback of estimated disturbance, or not. Black lines show steady state error without the feedback of estimated disturbance. Red lines show steady state error by proposed design method. They are compared in inside area less than edge of movable area because the stage was unstable in case of without the feedback of estimated disturbance. Fig. 13(a) and (c) show the comparison near the stage end. They were measured at $(50 \mathrm{~mm}, 50 \mathrm{~mm})$ and $(350 \mathrm{~mm}, 350 \mathrm{~mm})$. Fig. 13(b) shows the comparison at the center of movable area. It was measured at $(250 \mathrm{~mm}, 250 \mathrm{~mm})$. All results have shown that proposed design method has improved positioning accuracy. Amplitude of positioning error is less than $\pm 3 \mathrm{~nm}$.

4.4 Step response Finally, step response in vacuum environment is shown. Fig. 14 shows two step responses which are comparison of proposed design method and conventional one. They have been moved by same conditions of Table 2. Fig. 14(a) shows an overall step movement. They seem to be very similar response. Nevertheless, the difference can be found in Fig. 14(b) which shows close up of Fig. 14(a). There is

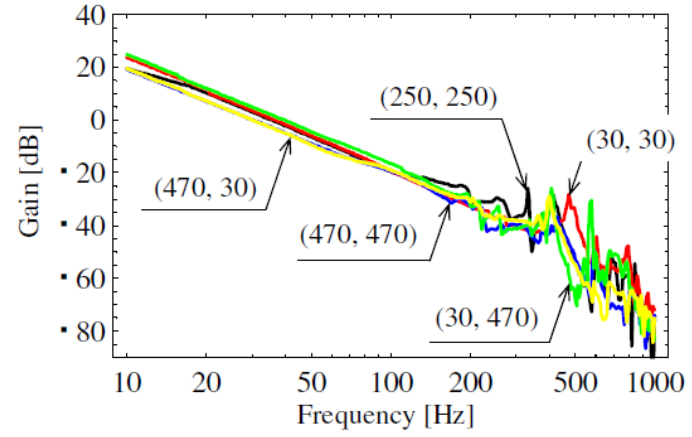

(a) Gain diagram

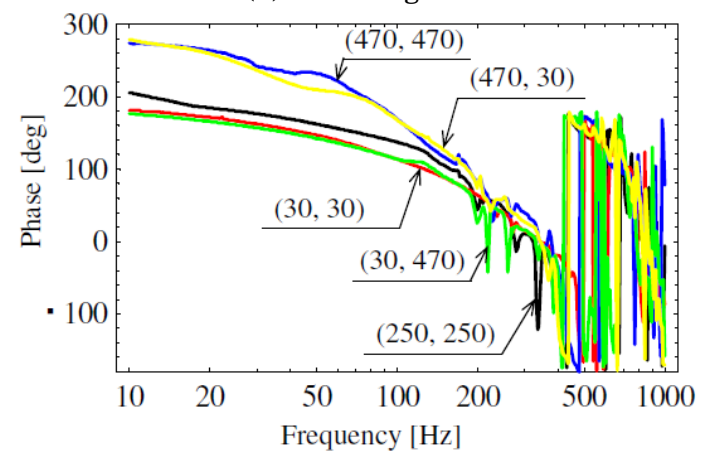

(b) Phase diagram

Figure 11: Open loop Bode diagram by proposed system at each position in the vacuum environment.

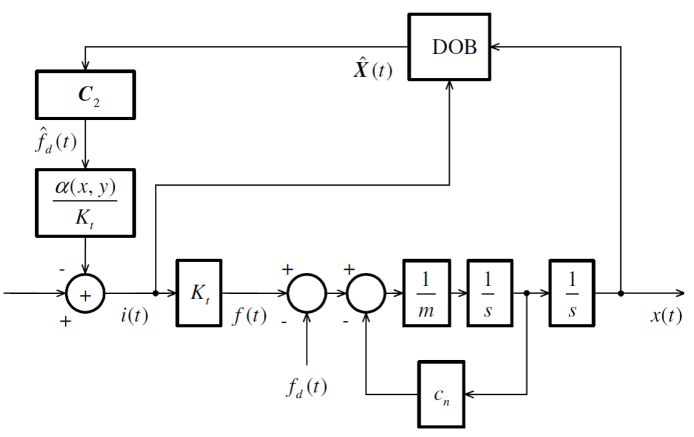

Figure 12: An equivalent block diagram to measure open loop Bode diagram. Disturbance is canceled by the feedback of estimated disturbance.

Table 2: Conditions of a step movement.

\begin{tabular}{lll}
\hline Condition & Value & Unit \\
\hline Moving distance & $60.0($ from 310 to 250$)$ & $\mathrm{mm}$ \\
Maximum acceleration & 1.96 & $\mathrm{~m} / \mathrm{s}^{2}$ \\
Maximum velocity & 0.3 & $\mathrm{~m} / \mathrm{s}$ \\
Pressure in a chamber & $2.31 \times 10^{-3}$ & $\mathrm{~Pa}$ \\
\hline
\end{tabular}

large overshoot value in case of moving by conventional method even though it was precisely adjusted in the atmosphere. On the other hand, a settling time, an overshoot value, and a steady state error were improved by proposed design method. These improvements have happened in whole movable area. 


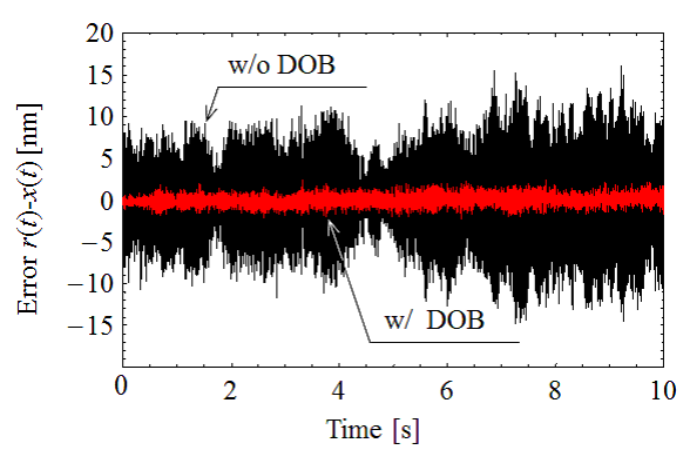

(a) Steady state error at $(50 \mathrm{~mm}, 50 \mathrm{~mm})$

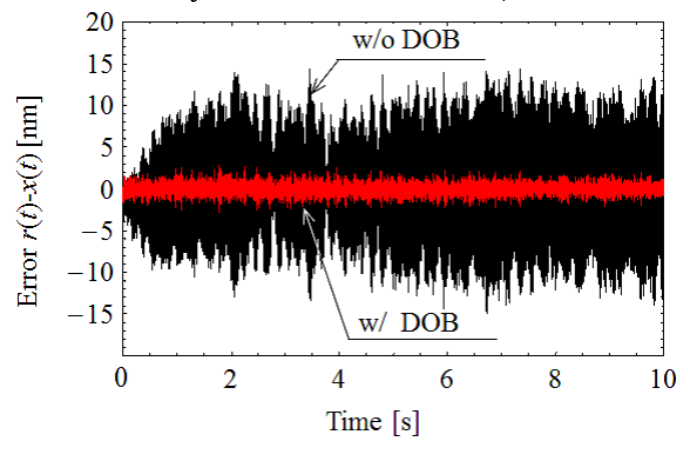

(b) Steady state error at $(250 \mathrm{~mm}, 250 \mathrm{~mm})$

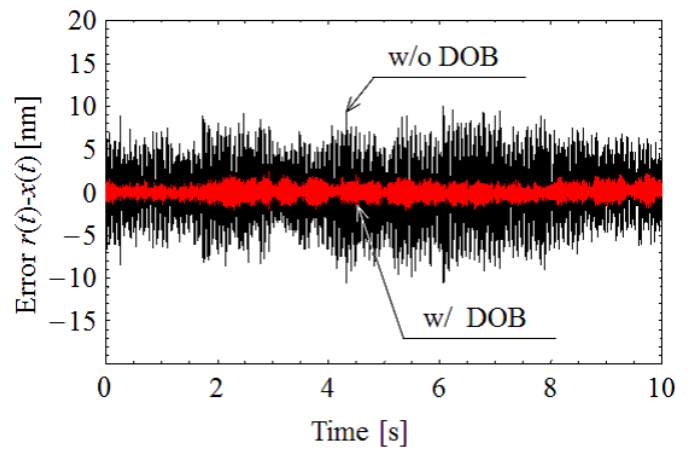

(c) Steady state error at $(350 \mathrm{~mm}, 350 \mathrm{~mm})$

Figure 13: Comparison of positioning accuracy by the feedback of estimated disturbance or not.

\section{Conclusion}

This paper proposed a control design method to precisely control the positioning XY stage which works in vacuum environment. Its method comprises the state feedback control and DOB considering position dependency of characteristics. This paper has shown that the feedback control of the estimated disturbance with PDP was effective by actual experiments even if there is the $\mathrm{XY}$ stage in vacuum environment. Then, this paper has shown that positioning accuracy has been less than \pm 3 $\mathrm{nm}$ by the experiments. It has also shown that the stage has been stable in whole movable area. As a conclusion, the proposed design method has yielded that it is possible to precisely control the positioning XY stage even though mechanical characteristic has been changing in vacuum environment.

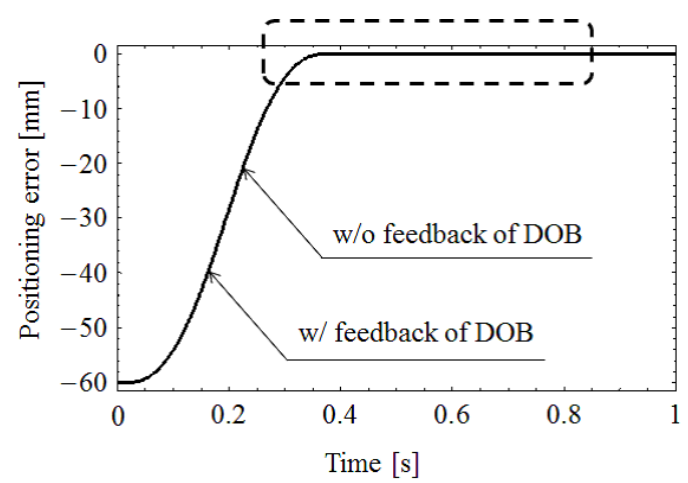

(a) Comparison of position error at step movement

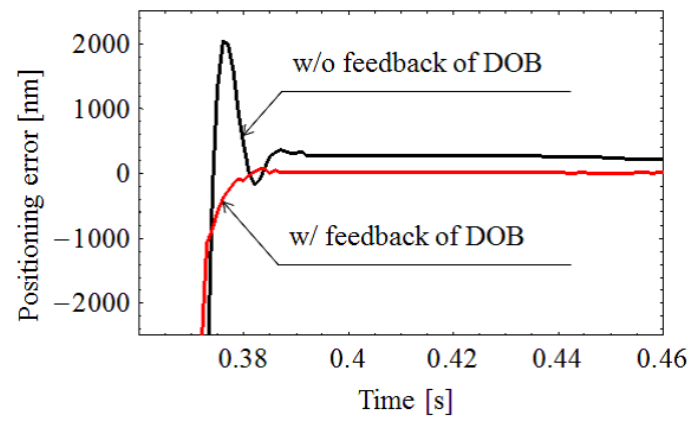

(b) Close-up of position error at step movement

Figure 14: An experiment of step movement in vacuum environment.

\section{References}

[1] ITRS 2013 Edition Table ORTC1: Executive Summary, International Technology Roadmap for Semiconductor, Semiconductor Industry Association, 2013.

[2] Tadashi Otaka, Hiroki Kawada, Chie Shishido and Masayuki Osaki, "Critical dimension measurement technology using CD-SEM”, Surface Analysis Techniques, vol.27, no.11, pp.636-641, 2006.

[3] Hiroo Kinohita, Munenori Kanai, Kimiyoshi Deguchi and Tadao Saito, "Air bearing guided high speed XY stage", Journal of the Japan Society for Precision Engineering, vol.52, no.10, pp.1713-1718, 1986.

[4] Yoshiyuki Tomita, Eiji Kojima, Shunichi Kawachi, Yasushi Koyanagawa and Seiji Ootsuka, "Development and applications of Sumitomo precision stage technologies for FPD process", Journal of the Japan Society for Precision Engineering, vol.78, no.2, pp.117-121, 2012.

[5] Keiichi Tanaka, "Feasibility of air levitated surface stage for lithography tool", Journal of Advanced Mechanical Design, Systems, and Manufacturing, vol.4, no.6, pp.1119-1131, 2014.

[6] Takumi Tsumura, Hayato Yoshioka, Hidenori Shinno and Hiroshi Sawano, "Magnetically preloaded aerostatic guideway for high speed nanometer positioning”, Journal of Advanced Mechanical Design, Systems, and Manufacturing, vol.8, no.4, pp.1-11, 2014. 
[7] Kazuhiro Yokoyama, Mizuki Saito, Hiraku Torinoumi, Toshimichi Moriwaki and Masato Aketagawa, "Improvement of positioning accuracy by real-time compensation of thermal elongation of ball-screw(1st report) - Principle of the compensation and influence of driving condition -", Journal of the Japan Society for Precision Engineering, vol.74, no.9, pp.931-936, 2008.

[8] Kenta Seki, Daisuke Noda and Makoto Iwasaki, "Highprecision positioning considering vibration and disturbance suppression in piezo-driven stage systems", Journal of Advanced Mechanical Design, Systems, and Manufacturing, vol.11, no.3, pp.1-10, 2017.

[9] Keisuke Umezawa, Yasuhiro Kakinuma, Seiichiro Katsura, Kohei Ohnishi, Tojiro Aoyama and Katsuhiko Yokoi, "Interaction mode control for the machine tool table with three linear servo motors", Journal of Advanced Mechanical Design, Systems, and Manufacturing, vol.2, no.4, pp.482.492, 2008.

[10] Daisuke Kurihara, Yasuhiro Kakinuma and Seiichiro Katsura, "Cutting force control applying sensorless cutting force monitoring method", Journal of Advanced Mechanical Design, Systems, and Manufacturing, vol.4, no.5, pp.955-965, 2010.

[11] Mohd Nazmin Maslan, Kaiji Sato, "Motion system design of a thin and compact linear switched reluctance motor with disposable-film mover", Journal of Advanced Mechanical Design, Systems, and Manufacturing, vol.12, no.1, pp.1-12, 2018.

[12] W. Chen, J. Yang, L. Guo and S. Li, "Disturbanceobserver-based control and related methods-An overview", IEEE Transactions on Industrial Electronics, vol.63, no.2, pp.1083-1095, 2016.

[13] D. Roy Choudhury, "Modern Control Engineering", pp.650-658, 2006, PHI Learning.

[14] Sergio Bittanti, Alan J. Laub, Jan C. Willems, "The Riccati Equation (Communications and Control Engineering)”, pp.243-262, 1991, Springer-Verlag Berlin Heidelberg.

[15] J. Ray Stagner, Gary C. Hart, "Application of the bilinear z-transform method to ground motion studies", Bulletin of the Seismological Society of America, vol.60, no.3, pp.809-817, 1970.

[16] Shreyas Fadnavis, "Image interpolation techniques in digital image processing: An overview", International Journal of Engineering Research and Applications, vol.4, no.10, pp.70-73, 2014.

[17] Prachi Rajarapollu, Vijay R Mankar, "Bicubic interpolation algorithm implementation for image appearance enhancement", International Journal of Computer Science and Technology, vol.8, no.2, pp.23-26, 2017.

[18] Jency Titus, Sebastian George, "A comparison study on different interpolation methods based on satellite images", International Journal of Engineering Research and Technology, vol.2, no.6, pp.82-85, 2013.

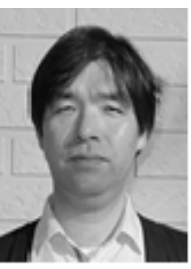

Morita Kazuhiro (Member) is senior engineer of Hitachi High Technologies Corporation at Ibaraki, Japan. He is lecturer of Electronic and Computer Engineering at National Institute of Technology, Ibaraki College in Ibaraki, Japan. He received his Ph.D. from Tokyo University of Agriculture and Technology in Japan. His research interests include precision positioning, damping control and its applications. He is member of IIAE.

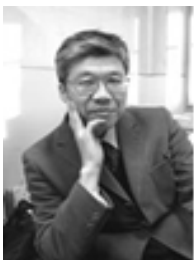

Wakui Shinji (Non-member) is professor of the Institute of Engineering at Tokyo University of Agriculture and Technology in Tokyo, Japan. He received his Ph.D. from Kanazawa University in 1993. His research interests precision positioning and its applications. 